\title{
On the calibration of rotational augmentation models for wind turbine load estimation by means of CFD simulations
}

\author{
Belen Soledad Burgos Tafur ${ }^{1,2} \cdot$ Elia Daniele ${ }^{1} \cdot$ Bernhard Stoevesandt $^{1} \cdot$ Philipp Thomas $^{1}$
}

Received: 16 September 2019 / Revised: 11 February 2020 / Accepted: 11 March 2020 / Published online: 25 April 2020

(C) The Author(s) 2020

\begin{abstract}
In this work the improved version of an engineering model which accounts for rotational augmentation effects by means of computational fluid dynamics (CFD) calibration is explored and discussed. Based on an analysis of the NREL Phase VI wind turbine, the novel modeling is presented, which uses as base line the formulation proposed by Chaviaropoulos and Hansen. The model is calibrated based on CFD simulations using OpenFOAM. The corresponding correction of the two dimensional polars is straightforward implemented within MoWiT, an in-house software for load calculation. The novel formulation results in improved lift and drag coefficients prediction in all considered cases, reducing the deviation with respect to the rotating CFD cases down to few percent. The optimal configuration including the correction for tip effects of Shen shows better agreements at the very tip of the blade. Furthermore the range of applicability for large wind turbine rotor blades based on a virtual $10 \mathrm{MW}$ rotor model is discussed.
\end{abstract}

\section{Introduction}

The Paris climate agreement in 2015 [1] aimed to reduce the greenhouse gas (GHG) and emissions in the biosphere, realizing the transition to a low-carbon economy. Wind energy sector has an important contribution to the fulfillment of these goals because of the targeted $28.5 \%$ of Europe's electricity demand by 2030 [2]. Added to the increasing demand for repowering of machines having reached the end of their lifetime [3], a number of challenges arose, among which enlarging the rotor blades of the wind turbines, while keeping the uncertainty about the related loads at a minimum. Robust tools such as aero-servo-elastic tools based on blade element momentum (BEM) theory are able to account for aerodynamic and structure interactions [4] with acceptable level of accuracy for most of the operating conditions. Within the last years, more sophisticated modeling based on high-fidelity aerodynamic approaches by the means of computational fluid dynamics (CFD) have emerged in parallel with a general development of aerodynamic modeling approaches [5]. The simplifica-

\section{Elia Daniele}

elia.daniele@iwes.fraunhofer.de

1 Fraunhofer IWES, Am Seedeich 45, 27572 Bremerhaven, Germany

2 Flensburg University of Applied Sciences, Wind Energy Technology Institute, Nordstraße, 224943 Flensburg, Germany tions made in BEM limit the accuracy of the obtained results. Therefore semi-empirical engineering models are incorporated in it for improving its accuracy. The empirical nature of some of the available engineering models, which have been developed based on rather small-size experiments, puts in doubt their general validity [4]. Nevertheless, the improvements demonstrated by CFD have served as a base-line for some researchers to define their models [6]. Thus, the use of CFD as a bridge to improve modeling in wind energy, seems to be the key point for deriving more sophisticated models which can be implemented in BEM. In this work the improved version of an engineering model which accounts for rotational augmentation (RAM) effects by means of CFD calibration is explored and discussed. The goal is to employ CFD simulations for the axial inflow of the NREL Phase VI wind turbine [6] to calibrate an alternative rotational augmentation model. This leads to a reduction in BEM deviations and it has been made ready for the IWES in-house load simulation library "Modelica for Wind Turbines" (MoWiT). Three-dimensional and rotational effects are the origin of the rotational augmentation phenomenon which is mainly characterized by the increment of the lift coefficient as compared with the two-dimensional case (infinite airfoil) due to the delay in the flow separation at higher angles of attack. The lack of modeling of the rotational augmentation phenomenon causes over or under prediction of aerodynamic loads on wind turbines [7]. Therefore, there is an imperative need of research in this field of study for improving 
load and power production prediction. Over the last years many authors have described several approaches to explain the occurrence of rotational augmentation on blade turbines. For Madsen and Rasmussen [8], the combination of centrifugal and Coriolis forces plus radial pressure gradients are the cause of rotational augmentation. Lindenburg [9] assures that Coriolis effects are dominant over centrifugal forces, relieving the adverse pressure gradients on the boundary layer and consequently delaying the separation [10]. For other researches $[11,12]$ the occurrence of the rotational augmentation is based on the blend of Coriolis, centrifugal forces and pressure gradients for the appearance of standing vortices on the blade suction surface. More recently, in Ref. [13], referring to large rotor size, it was observed that the root vortex system consist of counter-rotating trailing vortices which become stronger for the higher wind speed cases. In these same studies the ratio of the Coriolis to centrifugal forces weighted by the Rossby number was identified as capable of yielding information about the shear stress and boundary layer structure. In general, the effect of rotation is to increase the lift force in the separated flow region at inboard blade sections and decrease the lift force near the blade tip, but this part is still under research due to the inaccurate approach for tip corrections factors. However, the effect of rotation over the drag on the separated areas is still relative unknown. For instance, the work of Du and Selig [14] suggested that drag force decreases due to the separation moving towards the trailing edge, thus, shrinking the blade wake that reduces the sectional drag force on the rotating blade. Conversely, the work of other researchers, as Dowler et al. [10], indicated that the drag increases in a rotating system. The above mentioned different physical analysis led to alternative proposal for a semi-empirical modeling, to be later included within a load estimation tool. The investigations carried out by Snel [15] emphasized the analysis on boundary layer-equations for two-dimensional lift coefficient corrections, focusing on the ratio of local chord length to local radial location. Du and Selig [14] studied the integral threedimensional boundary layer including a tip speed ratio term and drag coefficient empirical correction. Chaviaropoulos and Hansen [16] developed a quasi-three-dimensional model based on the simplifications of the three-dimensional incompressible Navier-Stokes equations along the blade span-wise direction. The method described by Corrigan and Schillings [17] is characterized by the simplification of the boundary layer equations and was later evaluated by Tangler and Selig [18]. Bak et al. [19] proposed a model based on the analysis of the pressure distributions in a non-rotating and rotating blade. Dumitrescu et al. [20] introduced a correction model resulting from the simplification of the momentum integral equations (three-dimensional) for a general rotor blade. The model from Lindenburg [9] considered the centrifugal forces and the radial flow, the modified tip speed ratio and the local blade chord ratio as key parameter for the modification of the two-dimensional polars. Recently studies on large wind turbines blades show some interesting findings, as for instance, the work of Bangga et al. [21] indicating that the radial flow development at the inboard part of the blade creates a smaller size wake, inducing a reduction of the pressure level on the suction side, which in turn increases the lift coefficient. Until now, the mathematical "corrections" found in literature have been developed from experimental data, empirically or based on theoretical analyses. Some of these models correct both lift and drag, while others only the lift coefficient. The general formulation presented for the application of these corrections obeys to the following equations

$$
C_{L_{3 \mathrm{D}}}=C_{L_{2 \mathrm{D}}}+f_{L} \cdot \Delta C_{L},
$$$$
C_{D_{3 \mathrm{D}}}=C_{D_{2 \mathrm{D}}}+f_{D} \cdot \Delta C_{D} \text {, }
$$

where $\Delta C_{L}$ represents the difference between $C_{L_{2 \mathrm{D}}}$ (airfoil lift) and the inviscid lift coefficient, $\Delta C_{D}$ is the difference between $C_{D_{2 \mathrm{D}}}$ (airfoil drag) and the drag coefficient at zero angle of attack, $f_{L}$ and $f_{D}$ are correction factors which depend on the model, and $C_{L_{3 \mathrm{D}}}$ and $C_{D_{3 \mathrm{D}}}$ are the corrected lift and drag coefficients for three-dimensional effects.

For the sake of brevity hereby a thorough description of all considered models, from Snel [15] to Lindenburg [9], from Du and Selig [14] to Corrigan and Schillings [17], is avoided. Instead the focus on the formulation proposed by Chaviaropoulos and Hansen [16] is reported. The latter represents a quasi-three-dimensional approach based on viscous-inviscid interactions applied for laminar and turbulent flows. The model considers that three dimensional and rotational effects are strongly related to geometrical features of the blade such as the local chord to radial position ratio $c / r$ and the local twist angle $\theta$ of the blade. The maximum Mach number for this model is assumed to be 0.2 so then the flow can be studied as incompressible. The model has been derived from the quasi-three-dimensional incompressible Navier-Stokes equations in cylindrical coordinates taking the mean values and also making some assumptions for the mean values of the radial derivatives. A pressure correction algorithm is applied and the resulting pressure distribution around the airfoil is calculated, based on which lift and drag coefficients are deduced. Three-dimensional lift and drag coefficients are compared with the lift and drag from wind tunnel experiment, and finally three dimensional empirical relations have been deduced for the three dimensional correction. The corresponding correction factors for lift and drag coefficient are reported as

$$
\begin{aligned}
& f_{L}=a_{c h}\left(\frac{c}{r}\right)^{h_{c h}} \cos ^{n_{c h}} \theta, \\
& f_{D}=a_{c h}\left(\frac{c}{r}\right)^{h_{c h}} \cos ^{n_{c h}} \theta,
\end{aligned}
$$


where $a_{c h}=2.2, h_{c h}=1$ and $n_{c h}=4$ represent the constant, baseline parameters of this model [16]. According with this study, $c / r$ and $\theta$ does not influence the aerodynamic performance in the attached flow regime, but they play a very important role at higher angles of attack where the flow is separated. In this case the Coriolis force sucks mass from the recirculation region and redirects it to the radial direction reducing its volume. This results in a pressure drop along the suction side, and an increases blade loading [16]. Clearly, deducing the value of the aerodynamic forces from both experiments or simulations required the estimation of the angle of attack (AoA). In a rotating blade, this aspect is directly connected with the determination of the local inflow velocity in the rotor plane which is free of bound circulation (local undisturbed velocity) [22]. There are several methods for calculating the angle of attack at the rotor, thus, the analyses of this work are restricted to three proposals: average azimuthal technique (AAT) [23], three-point (3P) [24] and zero gamma (ZG) [22]. Since there is no common agreement within the research community on which method should represent the most suitable option, for the remaining of this work the indication of the AoA from threedimensional CFD simulation refers always to an averaged value of the results obtained by applying the three aforementioned methods. The possible application and validity of the proposed calibrated model when extended to larger size turbines is discussed by means of a reference to literature results concerning the AVATAR $10 \mathrm{MW}$ wind turbine virtual model based on the work described in Refs. [13,25,26]. The structure of the remaining part of the manuscript is as follows: a description of the CFD setup and its validation, together with the baseline modeling results compared to CFD, eevelopment of a model calibration based on the formulation of Chaviaropoulos and Hansen and its verification and validation, a concluding summary with outlooks.

\section{Methodology}

Figure 1 shows a flow-chart of the presented research. Standstill and rotating CFD simulations would be used to assess a hierarchy among the various modeling proposals available in the literature. By means of a comparison against selected experimental results the alternative model parameter are then derived and calibrated. Later this alternative is included into MoWiT.

The NREL Phase VI model wind turbine was used for conducting the numerical simulations of this work, since a wide range of experimental data was available for this wind small scale turbine model, which made it the perfect candidate for validating different type of simulations, in particular standstill and rotating ones. Differently from many other experimental wind turbine models where as well more complex inflow scenarios have been analyzed, e.g. Ref. [27], in the case of the NREL Phase VI experiment detailed pressure measurements at several span-wise sections allowed for aerodynamic force integration and validation. In this section general information about the specification of the NREL Phase VI is presented. Later on, a compact description of the CFD library-OpenFOAM is given, along with the procedure and details regarding to the set up for the performed simulations.

\subsection{Conducted simulations}

The NREL Phase VI wind turbine and the S809 airfoil were simulated using OpenFOAM library version $4 .{ }^{1}$ In order to reduce the number of simulations needed and allow for a broader verification of the results, both standstill and rotating cases were analyzed, in order to be able to compare multiple sections along the blade span-wise length by selecting different operating conditions for the two above mentioned cases. This circumstance lead to a fair agreement of the Reynolds number for the considered sections. For computing the twodimensional lift and drag sectional polars the blade has been pitched across a range of $78^{\circ}$, both in stall and feather direction, with $2^{\circ}$ spacing. Firstly, steady simulations at several inflow velocities are performed with standstill configuration. Table 1 contains the Reynolds numbers $(R e)$ along five spanwise positions corresponding to those for which experimental value are also available.

Standstill simulations provide information about the blade condition during the absence of rotation, although the finiteness effects at root and tip part of the blade does not allow a good agreement with two-dimensional airfoil simulations, available at $R e$ equal to $0.65 \times 10^{6}, 0.75 \times 10^{6}, 0.85 \times 10^{6}$ and $0.95 \times 10^{6}$. The simulation campaign for the rotating cases followed the same structure of that for the standstill configuration, except for the introduction of rotational effect by means of a source term in the momentum equation, as typically used in the advantageous case of single blade analysis. The related Reynolds number distribution along the spanwise direction is presented in Table 2 .

\subsection{Simulation setup}

The OpenFOAM CFD library is used for fluid dynamic simulations, while for grid generation, pre- and post-processing were done by means of IWES in house software [28]. The turbulence model employed is the $k-\omega$-SST [29] for its good stability in terms of convergence for the simulated cases. The initial values of boundary conditions are calculated based on what is reported in [30]. For all simulations the Reynolds-averaged Navier-Stokes equations (RANS) are

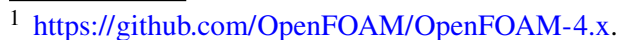




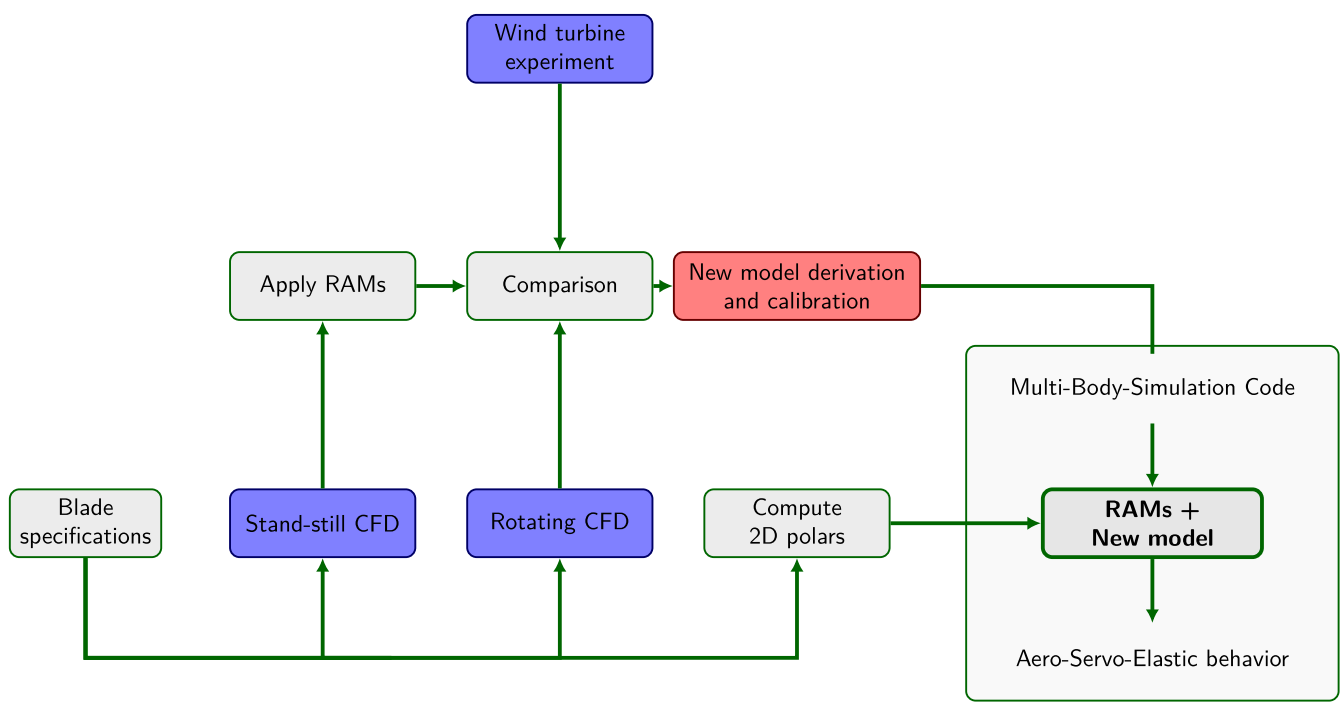

Fig. 1 Flow-chart of the presented research

Table 1 Reynolds numbers along five span-wise sections on the blade for the standstill simulations

\begin{tabular}{llllll}
\hline$r / R$ & \multicolumn{3}{c}{$\operatorname{Re}\left(\times 10^{6}\right)$} & $U_{\infty}=40$ & $U_{\infty}=50$ \\
\hline & $U_{\infty}=15$ & $U_{\infty}=20$ & $U_{\infty}=30$ & 1.89 & 2.37 \\
0.30 & 0.71 & 0.95 & 1.42 & 1.67 & 1.44 \\
0.47 & 0.63 & 0.84 & 1.25 & 1.22 & 1.80 \\
0.63 & 0.54 & 0.72 & 0.08 & 0.95 & 1.52 \\
0.95 & 0.46 & 0.61 & 0.72 & 1.27 \\
\hline
\end{tabular}

Unit of $U_{\infty}$ is $\mathrm{m} \mathrm{s}^{-1}$

Table 2 Reynolds numbers along five span sections on the blade for the rotating case

\begin{tabular}{llllll}
\hline$r / R$ & \multicolumn{5}{c}{$\operatorname{Re}\left(\times 10^{6}\right)$} \\
\cline { 2 - 6 } & $U_{\infty}=6$ & $U_{\infty}=7$ & $U_{\infty}=10$ & $U_{\infty}=15$ & $U_{\infty}=20$ \\
\hline 0.30 & 0.61 & 0.63 & 0.72 & 0.89 & 1.09 \\
0.47 & 0.78 & 0.795 & 0.85 & 0.97 & 1.11 \\
0.63 & 0.89 & 0.90 & 0.94 & 1.02 & 1.13 \\
0.80 & 0.94 & 0.95 & 0.97 & 1.03 & 1.11 \\
0.95 & 0.93 & 0.93 & 0.95 & 0.99 & 1.05 \\
\hline
\end{tabular}

Unit of $U_{\infty}$ is $\mathrm{m} \mathrm{s}^{-1}$

solved by means of the steady solver simpleFoam based on the SIMPLE algorithm [31], with a number of nonorthogonal correctors equal to 3 . The convective terms are discretized with a second order scheme, while for the diffusive terms a fully orthogonal scheme was used. Regarding the linear solvers, for the pressure equation a generalized geometric-algebraic multi-grid solver (GAMG) was used. For the equation of the velocity, the turbulent kinematic energy and its diffusion a smooth Gauss-Seidel solver was used. For the baseline two-dimensional simulations of the
S809 airfoil a modified parameter set of the baseline $k-\omega$-SST model was used to overcome the shortage in accuracy in predicting stall region. The HPC infrastructure of the University of Oldenburg [32] has been employed for all simulations.

\subsection{Validation of the simulations}

The CFD simulations were compared with the experimental results of NREL Phase VI [33] and S809 Airfoil [34]. In particular, for standstill cases, the sequence L3000ST0 is used, while for rotating case the sequence S0700000 is regarded. Although being RANS not the most suitable simulation approach for a configuration with extensive separated flow [35,36], it serves as a first step to measure the level of improvement a CFD driven parametrization may add to the baseline modeling. Another approximation resides in the choice of the turbulence model: Differently from the experiments, the presented simulation does not consider the presence of a laminar portion of the flow on blade and airfoil. This may impair the results for drag coefficient at low angle of attack as well as lift coefficient around the stall onset. In order to provide a qualitative assessment of the level of approxi- 


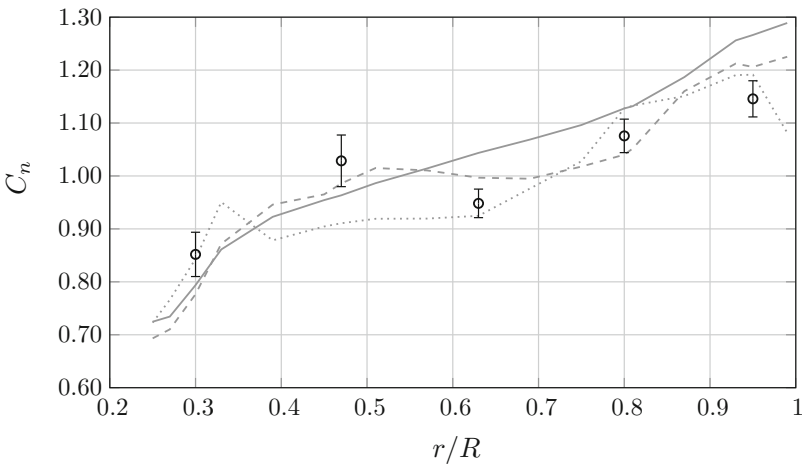

a Normal coefficient

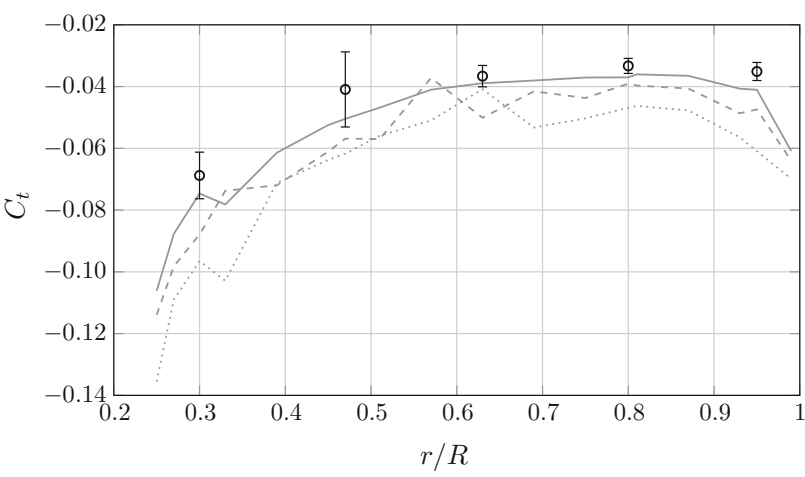

b Tangential coefficient

Fig. 2 Comparison of (a) normal and (b) tangential coefficients at 30 $\mathrm{m} \mathrm{s}^{-1}$ wind speed and $\beta=40^{\circ}$ between experiments (circles with error bar), RANS (-) and DES with (...) and without (- -) wake refinement. For standstill case

mation associated with the choice of a RANS modeling, in Fig. 2 the comparison of normal and tangential force coefficients along the blade span between experiment, RANS, and an alternative detached-eddy-simulation approach (DES) is presented.

These results indicate that DES simulations are more accurate in terms of $C_{n}$, see Fig. 2a, but not for $C_{t}$, as it does not improve the RANS solution, see Fig. 2b. Focusing on the inboard portion of the blade, standstill and rotating simulation are compared and validated in terms of pressure coefficient $C_{P}$ for different inflow velocities, and presented in Figs. 3 and 4. In both cases it exists a good agreement between experimental and numerical data.

Despite the possible validation for force and pressure coefficient, the proposal of an alternative model for rotational augmentation necessitates the capture of aerodynamic coefficient, thus, the AoA, or alternatively, the determination of the local velocity in the rotor plane without bound circulation [22], i.e. axial and angular induction factors. Several methods have been proposed for calculating the AoA, although studies of Guntur et al [37] reported the lack of a unique answer under different operating conditions.

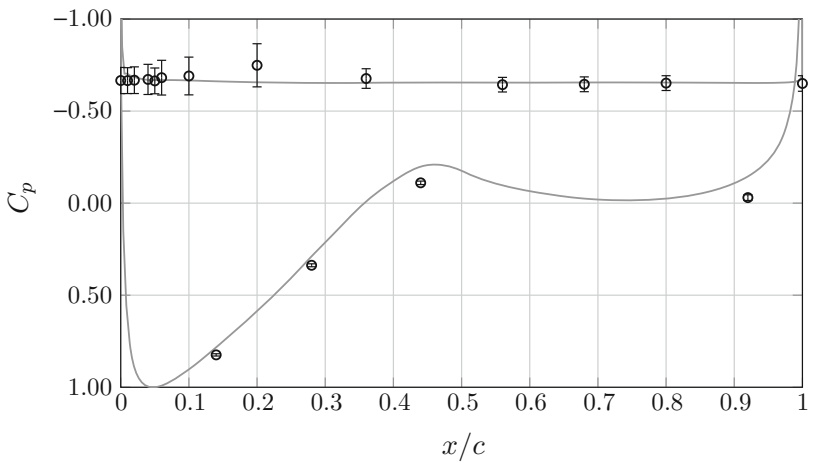

a $r / R=30 \%, \alpha=32.7^{\circ}$

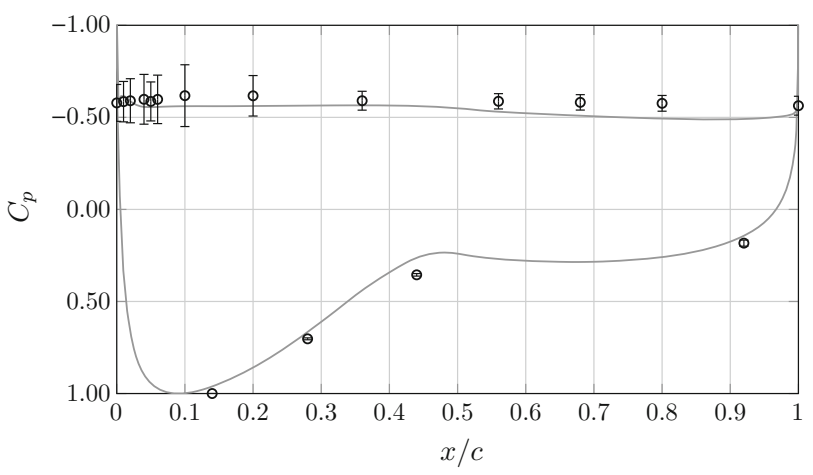

b $r / R=47 \%, \alpha=42.3^{\circ}$

Fig. 3 Pressure distributions at $30 \mathrm{~m} \mathrm{~s}^{-1}$ wind speed and $\beta=40^{\circ}$ at $r / R=30 \%$ and $47 \%$ : experiment (circles with error bar), RANS (-). For standstill configuration

Figure 5a shows a comparison of the AoA distribution along the blade span at four different inflow velocities $\left(7 \mathrm{~m} \mathrm{~s}^{-1}, 10 \mathrm{~m} \mathrm{~s}^{-1}, 15 \mathrm{~m} \mathrm{~s}^{-1}, 20 \mathrm{~m} \mathrm{~s}^{-1}\right)$. Results correspond to the mean value of three methods selected from literature (AAT, 3P, ZG, respectively after [22-24]). In the same figure with the solid black line is indicated the value of the stall angle of attack for the S809 airfoil. For an inflow wind velocity value equal to $7 \mathrm{~m} \mathrm{~s}^{-1}$ are observed AoA larger than the two-dimensional stall value from root up to $50 \%$ of the span, indicating the need for correction. Figure $5 \mathrm{~b}$ shows the comparison between two dimensional, stand still and rotating lift along the blade length, following the same schema derived from [21]. Results indicate the presence of augmentation effects at the inboard part of the blade, due to the increment of lift coefficients. Lift coefficient reduces as the radial position increases. The enhancement of $C_{L}$ is observed until $r / R=60 \%$. The calculated values of two dimensional $C_{L}$ at middle span-wise positions have good agreement with the rotating case. However, standstill polar coefficients show lower values than those for the two-dimensional case, probably due to large finiteness effects at root affecting the solution up to the mid-span. In areas close to the tip, the presence 


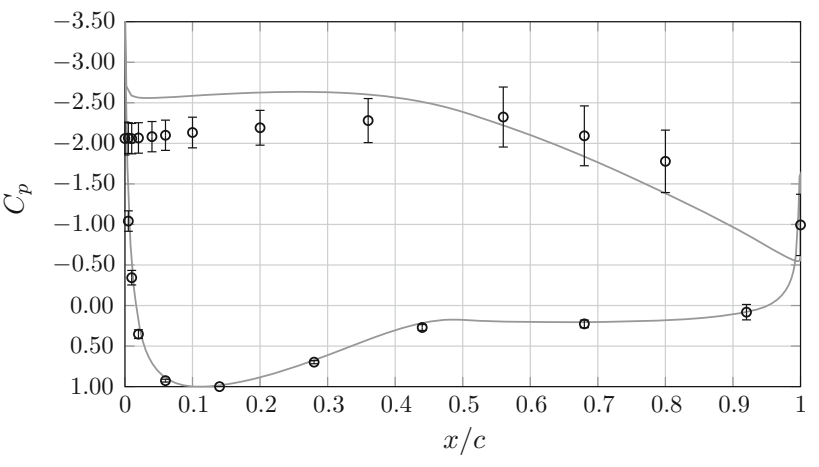

a $r / R=30 \%$

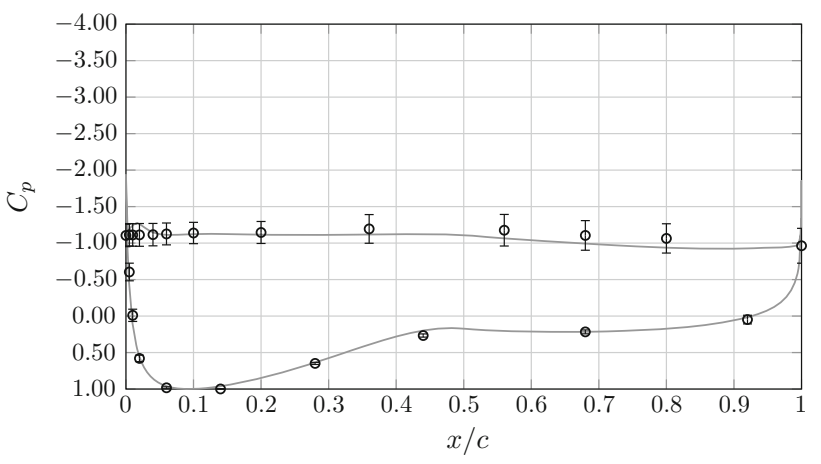

b $r / R=47 \%$

Fig. 4 Pressure distributions at $20 \mathrm{~m} \mathrm{~s}^{-1}$ wind speed and $\beta=-5^{\circ}$ at $r / R=30 \%$ and $47 \%$ : experiment (circles with error bar), RANS (-). For rotating configuration

of trailing vortices induces down-wash (tip effects), which causes a decrement in terms of lift coefficient.

\section{Results and discussion}

In this section the focus lies on the upper row of the flowchart reported in Fig. 1, i.e. applying the standard RAM and compare them against the rotating CFD cases in order to determine the development of the novel model parametrization.

\subsection{RAM verification}

In this section, two-dimensional polars are corrected for tip and root effects by means of Prandtl [38] and Shen [39] formulations. Afterwards, the polars are corrected for rotational augmentation effects using the available RAM described in the introduction. A schema of this procedure is presented in Fig. 6.

The two-dimensional polars corrected by finiteness and rotational augmentation effects are compared to those resulting from CFD for the rotating configuration. At the root region of the blade, two span-wise sections are analyzed at

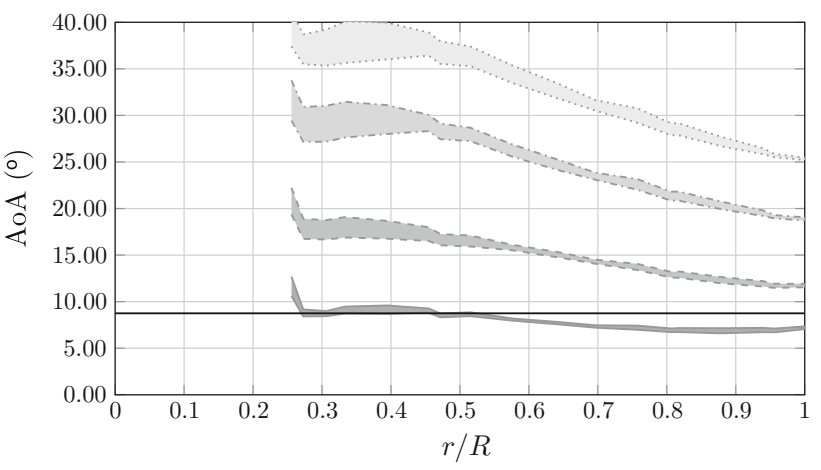

a Angle of attack

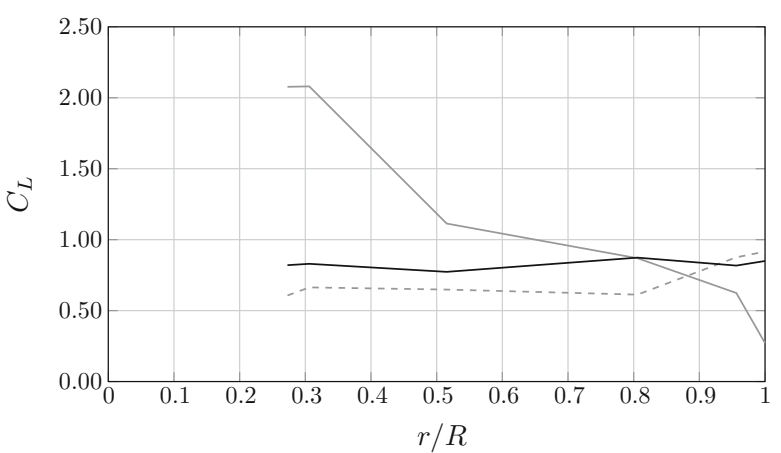

b Lift coefficient

Fig. 5 a AoA average value and standard deviation (as shaded area between the curves) distributions for $\beta=-5^{\circ}$ and different wind velocities compared to the stall angle for the two-dimensional case with an averaged Reynolds number value (-): $7 \mathrm{~m} \mathrm{~s}^{-1}(-), 10 \mathrm{~m} \mathrm{~s}^{-1}$ $(--), 15 \mathrm{~m} \mathrm{~s}^{-1}(-\cdot), 20 \mathrm{~m} \mathrm{~s}^{-1}(\cdots)$. b Comparison between lift coefficients along the blade for $15 \mathrm{~m} \mathrm{~s}^{-1}$ inflow wind velocity obtained from: two-dimensional airfoil simulation with Reynolds number varying according to the span-wise position (-), three-dimensional rotating case for $\beta=-5^{\circ}(-)$, three-dimensional standstill case (-)

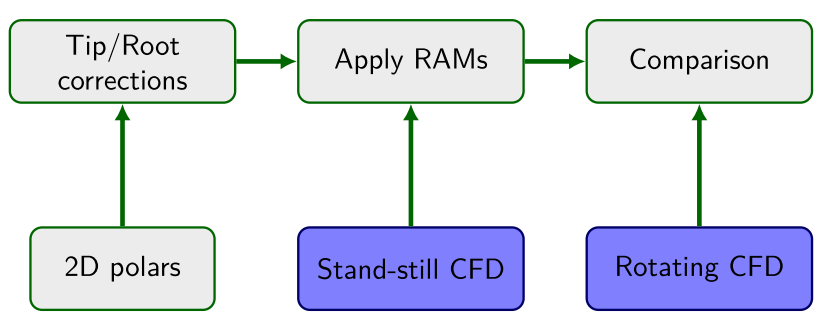

Fig. 6 Flow-chart of the RAM verification process

$r / R=33 \%$ and $39 \%$ for lift and drag. The two-dimensional polars are corrected by root effects using the formulation of Prandtl, whose modified proposal from Shen is used at tip. Subsequently, these finiteness-corrected polars are further manipulated to account for rotational augmentation effects using the following models: Du and Selig [14] (DS), Chaviaropoulos and Hansen [16] (CH), Snel [15] and Lindenburg [9] (SL). Considering the case with inflow wind velocity equal to $7 \mathrm{~m} \mathrm{~s}^{-1}$ and rotational speed of the rotor 


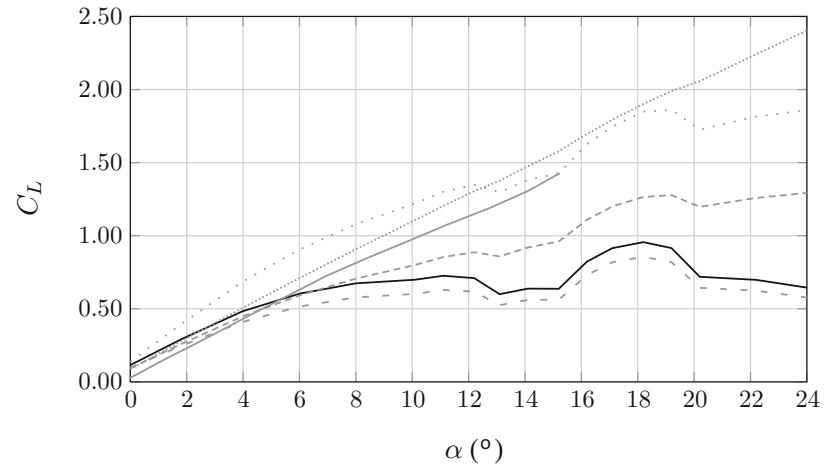

a $r / R=33 \%$

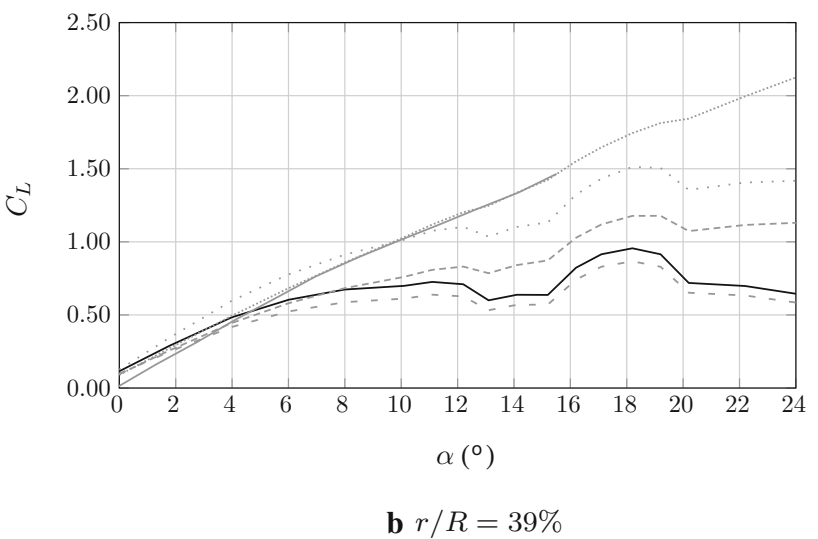

Fig. 7 Comparison of lift coefficient for the rotating CFD case and two dimensional polars after the application of root correction and RAM at two span-wise positions: a 33\%, b 39\%. Legend for both graphics: two-dimensional case (-), three-dimensional case (-), Prandtl-corrected two-dimensional case (- -), Prandtl-DS-corrected two-dimensional case (--), Prandtl-SL-corrected two-dimensional case (. .), Prandtl-CHcorrected two-dimensional case (..)

kept at 72 rpm, Figs. 7, 8 and 9 report the lift polars at blade root and tip.

Figure 7 illustrates the inboard sections. The DS model does not sufficiently correct the polars after $5^{\circ}$ AoA. A similar behavior is exhibited by the SL model, although, in this region both models over-predict the lift in the linear part. The $\mathrm{CH}$ model is in good agreement for both considered inboard sections.

The correction for finiteness at tip differs depending on the adoption of the model. For this reason for the last section within the tip portion of the blade both the model of Prandtl and the model of Shen are used prior to the application of the RAM to improve the assessment. The formulation of Prandtl shows large reduction of lift in both the linear range and after stall. This results in deviations from the result of the rotating CFD case of $60 \%$ and $40 \%$ for, respectively, the DS and $\mathrm{CH}$ model in the linear range, while after stall the deviation reach $90 \%$ with DS and $45 \%$ with $\mathrm{CH}$, see Figs. 8a and $9 \mathrm{a}$. The behavior of the corrected polars changed when

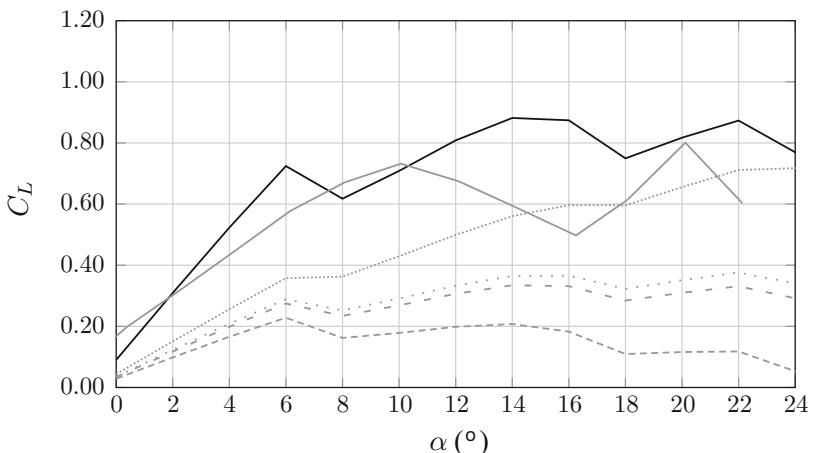

a Tip correction type: Prandtl

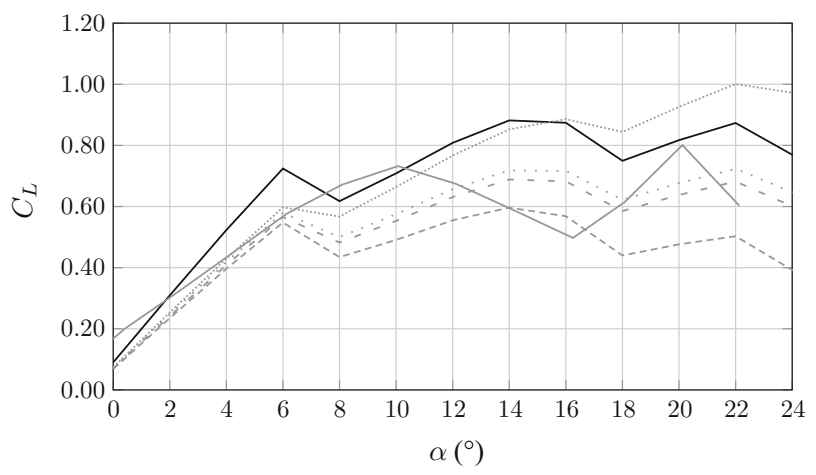

b Tip correction type: Shen

Fig. 8 Comparison of lift coefficient for the rotating CFD case and twodimensional polars after the application of tip corrections and RAM at $r / R=95 \%$. Legend for both graphics: two-dimensional case (-), threedimensional case (-), tip-corrected two-dimensional case (- -), tip-DScorrected two-dimensional case (--), tip-SL-corrected two-dimensional case $(\cdot)$, tip-CH-corrected two-dimensional case $(\cdot)$. Tip correction type is indicated in the sub-caption

the formulation of Shen in combination with the $\mathrm{CH}$ model is applied, as demonstrated in Figs. $8 \mathrm{~b}$ and 9b. Results show good agreement in the linear range and deviations about $20 \%$ after stall.

Based on the results discussed quantitatively above non of the considered RAM alternatives is able to capture the features of the rotating polars along the entire blade span. Nevertheless, the $\mathrm{CH}$ model showed the smallest deviations among all other models.

\subsection{Derivation of novel parametrization for RAM}

Having identified a the good agreement between two- and three-dimensional polars by means of the adoption of the $\mathrm{CH}$ model in the previous section, a novel parametrization for RAM based on the $\mathrm{CH}$ formulation is proposed, aiming to extend its validity along the entire length of the blade, independently of the operating condition, being the latter for the NREL Phase VI related to the inflow wind velocity. 


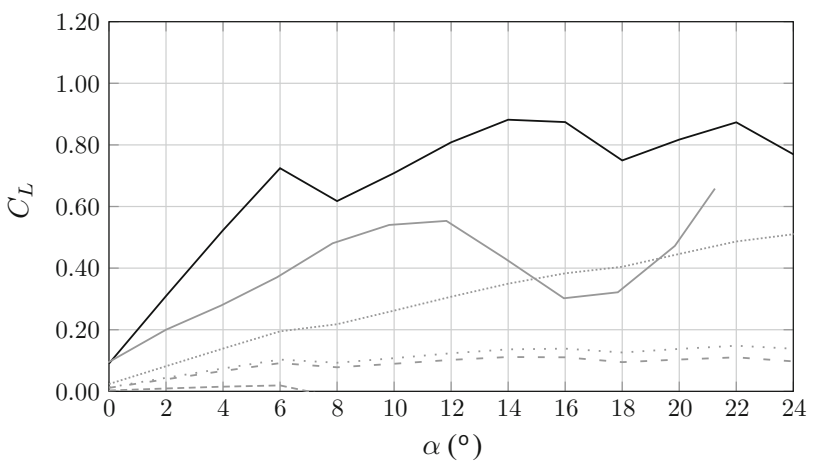

a Tip correction type: Prandtl

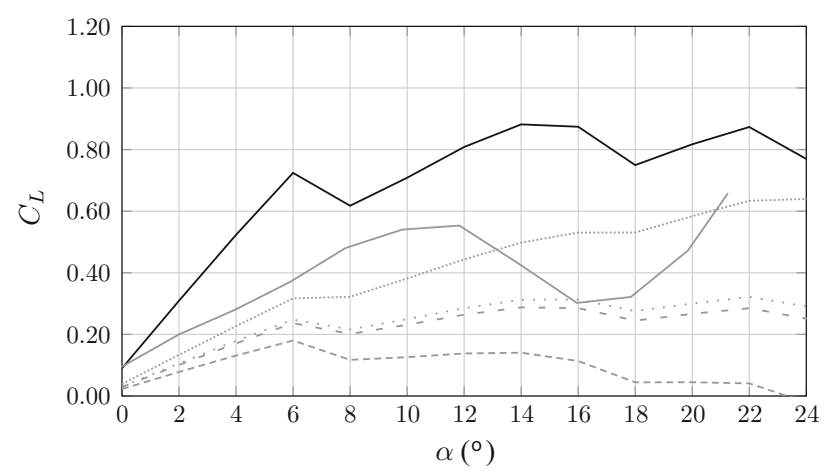

b Tip correction type: Shen

Fig. 9 Comparison of lift coefficient for rotating CFD and twodimensional polars after the application of tip corrections and RAM at $r / R=99 \%$. Legend for both graphics: two-dimensional case $(-)$, three-dimensional case (-), tip-corrected two-dimensional case (-), tip-DS-corrected two-dimensional case (--), tip-SL-corrected twodimensional case ( $\cdot$.), tip-CH-corrected two-dimensional case ( $\cdot)$. Tip correction type is indicated in the sub-caption

As it was described in the introduction, the $\mathrm{CH}$ model provides corrections for lift and drag coefficients based on the value of the variables $c / r$ and $\phi$, and the constant parameters $a_{c h}, h_{c h}, n_{c h}$, see Eqs. (3) and (4).

The two-dimensional polars are corrected to fit to the CFD rotating polars by means of the baseline $\mathrm{CH}$ and nonlinear least squares method. While $h_{c h}$ and $n_{c h}$ are kept equal to the baseline literature values, an alternative value for $a_{n}$ is derived based on the minimization of the deviation between two and three-dimensional polars. Since RAM applies to rotating blade whose finiteness is not included in the two-dimensional polars, the same procedure is applied after correcting the two-dimensional polars for tip and root effects as well. Figure 10 shows an example of the fitting process for determining the optimal $a_{n}$ value. In this specific case the two-dimensional polars without tip and root corrections are reported, with $h_{c h}=1, n_{c h}=4$, and $15 \mathrm{~m} \mathrm{~s}^{-1}$ inflow velocity at a $33 \%$ span-wise location.

The value calculated for $a_{n}$ is 1.2 (Fig. 10a) and 2.2 (Fig. 10b) for, respectively the case of lift and drag coef-

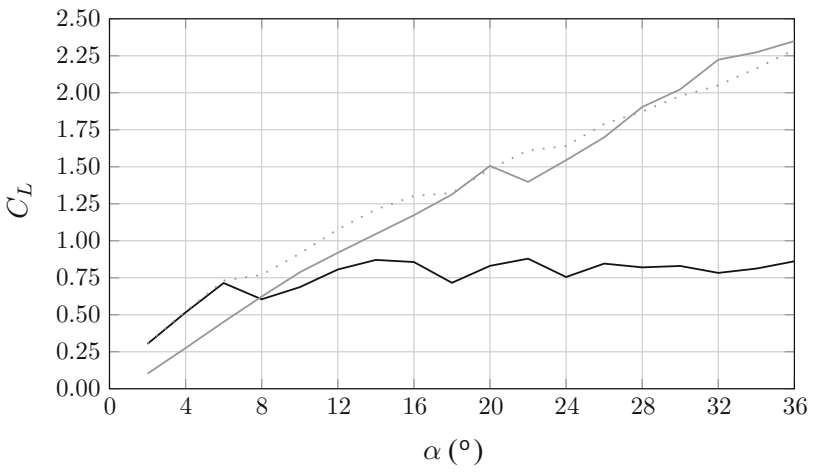

a Lift polar

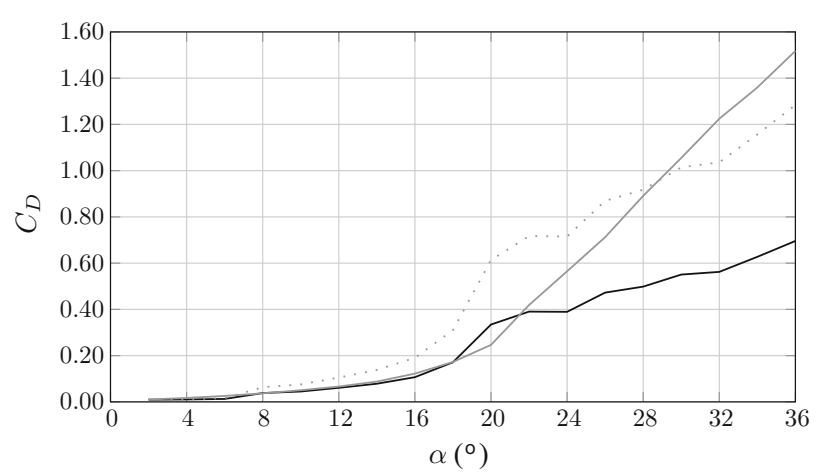

b Drag polar

Fig. 10 Fitting function for $a$ using $h_{c h}=1$ and $n_{c h}=4$, a lift polar, b drag polar at $15 \mathrm{~m} \mathrm{~s}^{-1}$ inflow speed, and $r / R=33 \%$. Legend for both graphics: two-dimensional case (-), three-dimensional case (-), fitting procedure result $($. )

ficient, for which the calculated, optimal $a_{n}$ equals the value from the literature [16].

An analogous investigation is applied for all the conditions indicated in Table 2, resulting in a distribution of optimal values for $a_{n}$ depending on the inflow velocity and the spanwise position, as showed in Fig. 11.

Regarding the lift coefficient, $a_{n}$ values that minimize the deviation between two-dimensional and three-dimensional polars vary strongly with the span-wise position. Three different regions along the blade length are identified: $25-40 \%$, $40-80 \%$, and from $80 \%$ to the tip. After $10 \mathrm{~m} \mathrm{~s}^{-1}$, i.e. as the turbine starts to be stall-regulated, the behavior of $a_{n}$ changes abruptly, and it is mainly due to the fact that the flow becomes extremely unstable and separated. Accordingly, after rated wind speed one single tendency is observed throughout the entire blade length.

The value of $a_{n}$ presents also a dependency on the inflow velocity value even when the flow is mostly non-separated along the blade, namely between 6 and $10 \mathrm{~m} \mathrm{~s}^{-1}$. Within the inboard region from 25 to $40 \% r / R$, no significant influence of the inflow velocity is noticed. Above $40 \% r / R$ the behavior of $a_{n}$ changes, reaching its inversion point towards the tip, as the inflow velocity increases. The last portion of the 


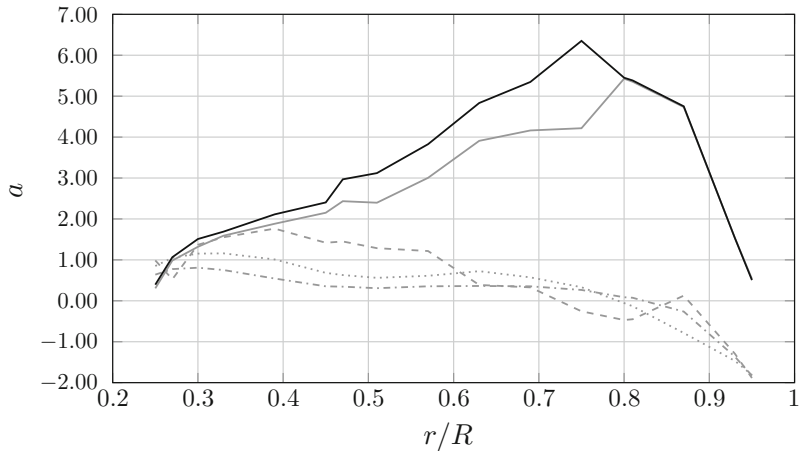

a Lift polar

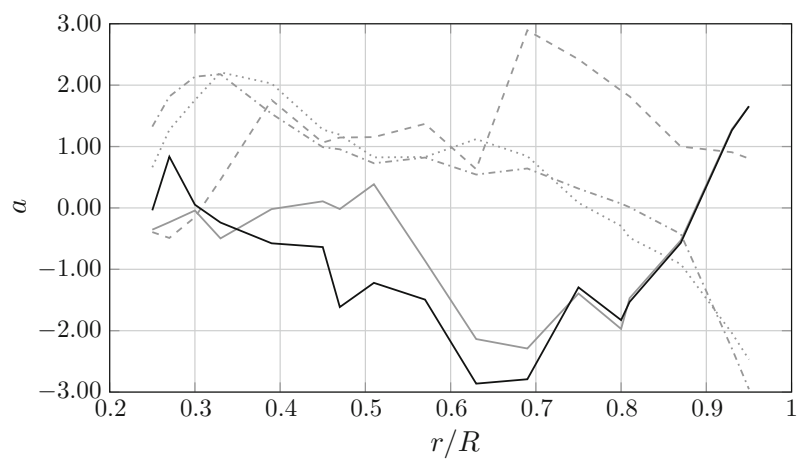

b Drag polar

Fig. 11 Results of optimal $a_{n}$ without tip and root corrections for a lift and $\mathbf{b}$ drag coefficient polar at different wind speeds. Legend for both graphics: $6 \mathrm{~m} \mathrm{~s}^{-1}(-), 7 \mathrm{~m} \mathrm{~s}^{-1}(-), 10 \mathrm{~m} \mathrm{~s}^{-1}(--), 15 \mathrm{~m} \mathrm{~s}^{-1}(\cdot)$, $20 \mathrm{~m} \mathrm{~s}^{-1}(-\cdot-)$

blade also presents some dependency on inflow velocity as it is shown in Fig. 11a.

It is more challenging to identify a pattern for the optimal values of $a_{n}$ for the drag coefficient. However, here also the $a_{n}$ values seem to depend on the span-wise position and the inflow velocity. Two regions are identified: from $25 \%$ until $70 \%$ and from $70 \%$ until tip. Regarding the inflow velocity there is a dependency of the calculation of $a_{n}$ when the flow is non-separated, and above the rated velocity, one tendency for $a_{n}$ emerges, see Fig. $11 \mathrm{~b}$.

In order to show the effects of tip and root corrections on the behavior of the optimal $a_{n}$, the results of the fitting procedure based on the lift coefficient after the application of Prandtl and Shen models are reported, respectively, in Fig. 12a, b.

The effect of Prandtl correction is more evident at the tip, where the optimal $a_{n}$ behaves completely different from what observed in Fig. 11a. The dependency of $a_{n}$ on the inflow velocity is kept similar as it was before the application of Prandtl model. The model of Shen provides variations at the tip region at both low and high inflow velocity values, leading to larger values for the optimal $a_{n}$ compared to those seen in Fig. 11a.

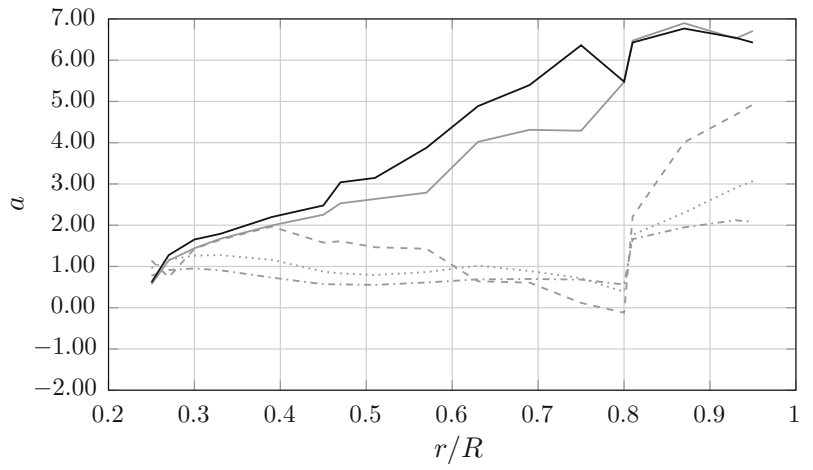

a Prandtl

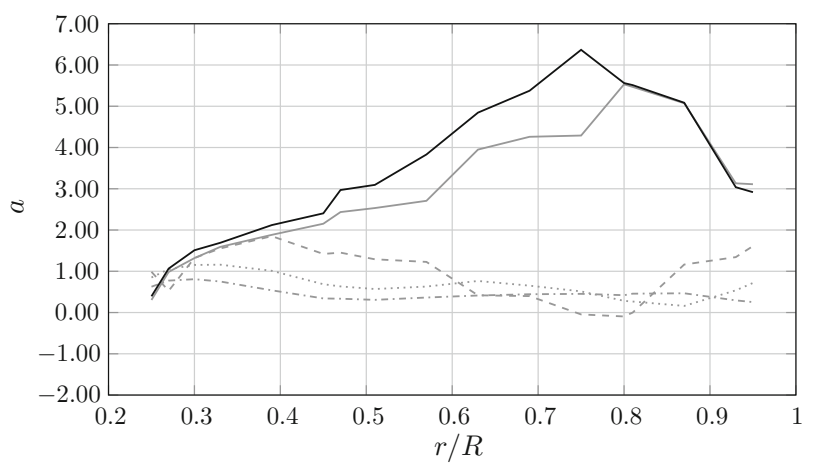

b Shen

Fig. 12 Results of the optimal $a_{n}$ in conjunction with the application of the correction models of $\mathbf{a}$ Prandtl and $\mathbf{b}$ Shen for the lift coefficient at different wind speeds. Legend for both graphics: $6 \mathrm{~m} \mathrm{~s}^{-1}(-), 7 \mathrm{~m} \mathrm{~s}^{-1}$ $(-), 10 \mathrm{~m} \mathrm{~s}^{-1}(--), 15 \mathrm{~m} \mathrm{~s}^{-1}(\cdot), 20 \mathrm{~m} \mathrm{~s}^{-1}(-\cdot-)$. Tip correction type is indicated in the sub-caption

\subsubsection{Alternative parameters' formulation}

In this subsection is presented the formulation for an optimal $a_{n}$ for the lift coefficient obtained in conjunction with the application of the Shen tip correction model, that depends on the span-wise position and the inflow velocity value. Being the NREL Phase VI turbine model a stall-regulated machine, the distinction based on the operating condition is solely referred to the inflow velocity value. Based on the results of the previous section two types of parameters' formulations are proposed for the lift coefficient below and above an inflow velocity equal to $10 \mathrm{~m} \mathrm{~s}^{-1}$. For low inflow velocities, Eqs. (5), (6) and (7) represent the alternative parameters' formulation of $a_{n}$ for the three distinct regions for the lift coefficient. The value of the fitting parameters $b_{1 L}, b_{2 L}, b_{3 L}, c_{1 L}, c_{2 L}, c_{3 L}, d_{2 L}, d_{3 L}$ and $e_{2 L}, e_{3 L}$ are reported in Table 3 . These are obtained by applying the fitting procedure that minimize the deviation of the polars using Eqs. (5), (6) and (7).

$a_{n 1 L}=b_{1 L} \cdot \frac{r}{R}+c_{1 L}$, 
Table 3 Coefficients used for the optimal $a_{n}$ alternative parametric formulation for lift at low inflow velocities $\left(6-10 \mathrm{~m} \mathrm{~s}^{-1}\right)$, in conjunction with the application of the Shen correction model at the tip

\begin{tabular}{llll}
\hline Two-dimensional polars & $r / R=0-40 \%$ & $r / R=40 \%-80 \%$ & $r / R=80 \%-100 \%$ \\
\hline Shen corrected & $b_{1 L}=7.8$ & $b_{2 L}=-4.13$ & $b_{3 L}=7.1$ \\
& & $c_{2 L}=36.6$ & $c_{3 L}=-59.5$ \\
& $c_{1 L}=-1.1$ & $d_{2 L}=1.55$ & $d_{3 L}=-7.1$ \\
& & $e_{2 L}=-11.78$ & $e_{3 L}=91.91$ \\
\hline
\end{tabular}

Table 4 Coefficients used for the optimal $a_{n}$ alternative parametric formulation for lift at high inflow velocities $\left(10-20 \mathrm{~m} \mathrm{~s}^{-1}\right)$, in conjunction with the application of the Shen correction model at the tip

\begin{tabular}{ll}
\hline Two-dimensional polars & $r / R=0-100 \%$ \\
\hline Shen corrected & $b_{1 L}=0.68$ \\
& $c_{1 L}=1.09$ \\
\hline
\end{tabular}

$a_{n 2 L}=\left(b_{2 L} \cdot U_{\infty}+c_{2 L}\right) \cdot \frac{r}{R}+\left(d_{2 L} \cdot U_{\infty}+e_{2 L}\right)$,

$a_{n 3 L}=\left(b_{3 L} \cdot U_{\infty}+c_{3 L}\right) \cdot \frac{r}{R}+\left(d_{3 L} \cdot U_{\infty}+e_{3 L}\right)$.

The analysis performed for inflow velocities above $10 \mathrm{~m} \mathrm{~s}^{-1}$ led to Eq. (8). Table 4 shows the coefficients obtained by means of the fitting procedure that minimize the deviation of the polars using the above mentioned equation.

$a_{n 1 L}^{\prime}=b_{1 L}^{\prime} \cdot \frac{r}{R}+c_{1 L}^{\prime}$

\subsubsection{Assessment}

The identified optimal configuration is assessed by comparing it against the results of the rotating CFD simulation cases used as reference. The two-dimensional polars are corrected by both the baseline and the alternative parametric $\mathrm{CH}$ formulation proposed by the authors.

The accuracy of the alternative parametric formulation is investigated by comparing the optimal configurations with (models $a_{n_{\text {Prandtl }}}$ and $a_{n_{\text {Shen }}}$ ) and without (model $a_{n}$ ) tip and root corrections. Three sections along the blade are studied: $r / R=33 \%, 69 \%$ and $95 \%$. Two inflow velocity values are considered, e.g. $U_{\infty}=7 \mathrm{~m} \mathrm{~s}^{-1}$ and $U_{\infty}=20 \mathrm{~m} \mathrm{~s}^{-1}$, in order to assess the model performance at both low and high inflow velocities.

Regarding to lift coefficient, at $r / R=33 \%$ the alternative parametric model outperforms the original one, see Fig. 13).

For example, at $15^{\circ}$ AoA the baseline formulation of $\mathrm{CH}$ shows deviations of $15 \%$ and $40 \%$ at $U_{\infty}=7 \mathrm{~m} \mathrm{~s}^{-1}$ $U_{\infty}=20 \mathrm{~m} \mathrm{~s}^{-1}$, respectively. Conversely, the alternative parametric formulation shows improvements, following closely the rotating polars with higher accuracy along the
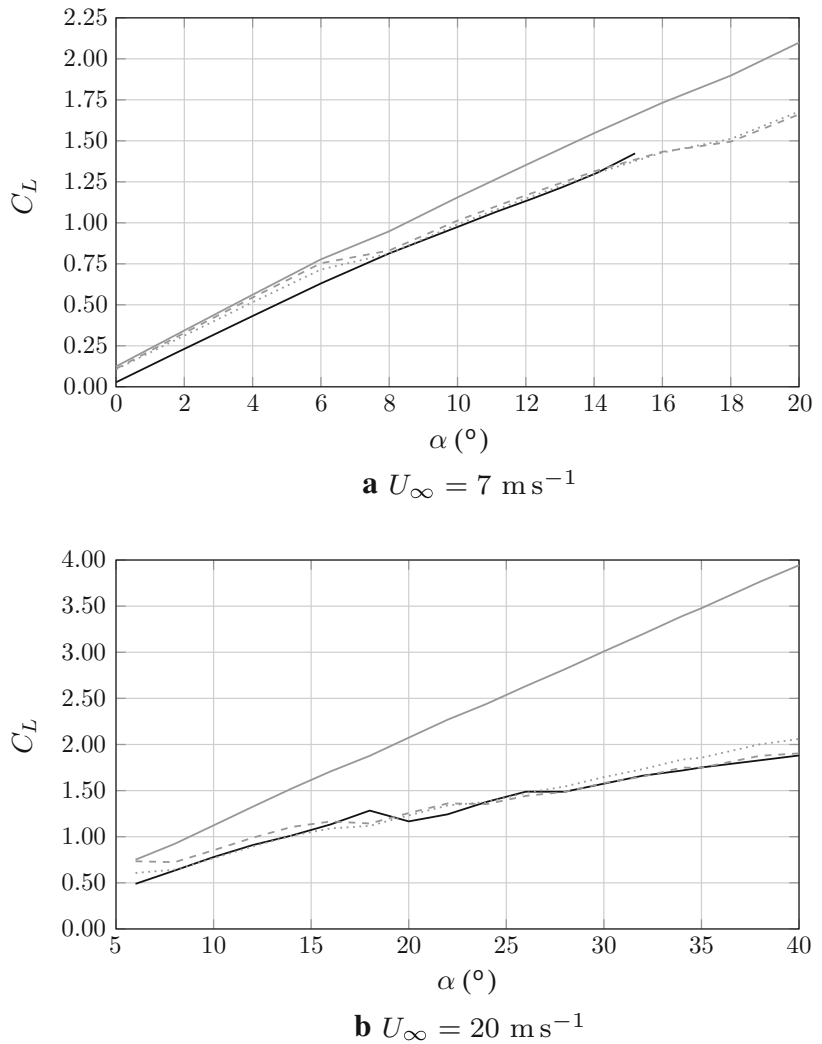

Fig. 13 Lift corrected by augmentation effects at $r / R=33 \%$. Computations are shown for the proposed alternative parametric formulation, the baseline formulation of $\mathrm{CH}$ and the CFD rotating results for two different wind velocity values. Legend for both graphics: threedimensional CFD rotating case (-), $\mathrm{CH}$-corrected two-dimensional case (-), fitting-model-corrected two-dimensional case (- -), fitting-modelPrandtl-corrected two-dimensional case ( $\cdot *)$. The wind velocity value is indicated in the sub-caption

entire curve. At $20 \mathrm{~m} \mathrm{~s}^{-1}$ inflow velocity (Fig. 13b), the model based on Prandtl correction for root effects further improves the results of about $5 \%$ below $15^{\circ}$ AoA, while for larger AoA the model with $a_{n}$ seems better. At lower inflow velocity values (Fig. 13a) the $a_{n \text { Prandtl }}$ and $a_{n}$ variants present similar results.

In the tip region, the formulation based on the Shen corrections for tip effect (model $a_{n_{\text {Shen }}}$ ) is also presented. At low inflow speeds, the model $a_{\text {Prandtl }}$ exhibits excellent agreements with the rotating CFD polars (Fig. 14a). As the 


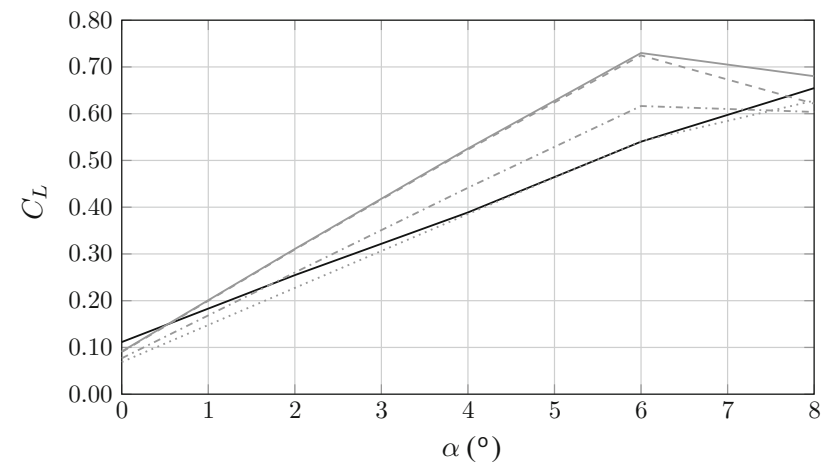

a $U_{\infty}=7 \mathrm{~ms}^{-1}$

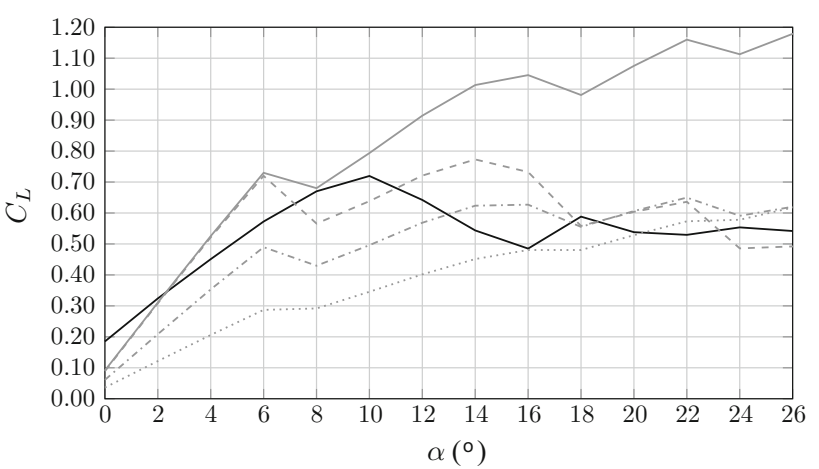

b $U_{\infty}=20 \mathrm{~ms}^{-1}$

Fig. 14 Lift corrected by augmentation effects at $r / R=95 \%$. Computations are shown for the proposed alternative parametric formulation, the baseline formulation of $\mathrm{CH}$ and the CFD rotating results for two different wind speed values. Legend for both graphics: three-dimensional CFD rotating case (-), CH-corrected twodimensional case (-), fitting-model-corrected two-dimensional case ($-)$, fitting-model-Prandtl-corrected two-dimensional case (..), fittingmodel-Shen-corrected two-dimensional case (---). The wind velocity value is indicated in the sub-caption

inflow velocity increases the $a_{n_{\text {Shen }}}$ and $a_{n}$ variants show better results, and about $20 \%$ deviation is seen in both cases (Fig. 14b).

The alternative parametric formulation of the $\mathrm{CH}$ model results in improved lift and drag coefficients prediction in all cases. The optimal configuration including the correction for tip effects of Shen shows better agreements at the very tip of the blade. Slightly improvements were seen at the root when the configuration is accounting for the model of Prandtl.

\subsection{Extension to large wind turbine rotor-blade scenarios}

In this section the extension of the proposed modification is discussed based on the studies reported in Refs. [13,25,26] for the case of a $10 \mathrm{MW}$ wind turbine rotor virtual model developed within the framework of the AVATAR EU project [40].

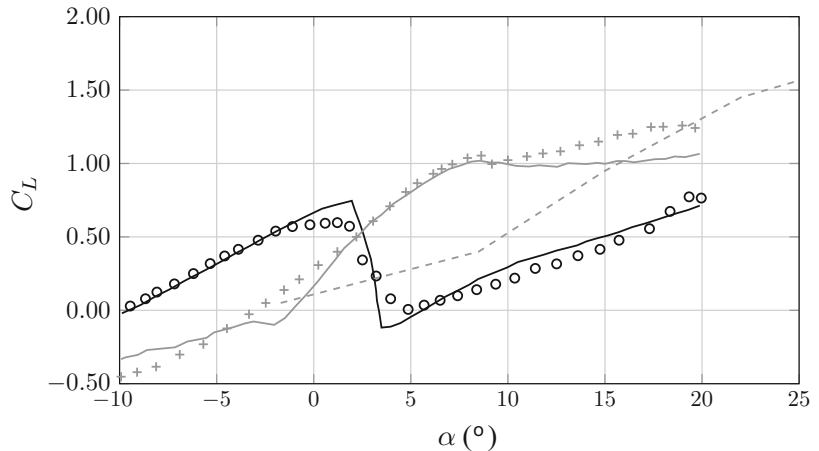

Fig. 15 Lift coefficient for the airfoil DU-00-W-401. Experimental and panel-code results from Ref. [42] for a Reynolds number equal to $3 \times$ $10^{6}$. CFD results from Ref. [25] for the same airfoil located at the $25 \%$ of the blade length for the AVATAR rotor. Legend: experimental result with free transition $(+)$, experimental result for forced transition at $2 \%$ suction side and $10 \%$ pressure side (o), numerical result from panel-code simulation with free transition (-), numerical result from panel-code simulation with forced transition at $2 \%$ suction side and $10 \%$ pressure side (-), CFD unsteady RANS from Ref. [25] for a comparable airfoil section having the maximum relative thickness equal to $40 \%$ and located at the $25 \%$ of the blade length for the AVATAR rotor (- -)

In Fig. 15 are reported the experimental and numerical results for a characteristics inboard section of the AVATAR rotor, where rotational augmentation effects are relevant. The related airfoil type is the DU-00-W-401, developed at TU Delft in The Netherlands, with the aim of having a reduced sensitivity to the roughness height for moderate to large thickness and large Reynolds number. Analogous airfoil families have been developed for low Reynolds number as well, e.g. in Ref. [41]. The impact of inflow turbulence and/or surface roughness is well represented by means of the CFD results available in same figure as computed in Ref. [25]. The high value for the airfoil maximum relative thickness introduce a pronounced difference in the lift coefficient polar between the free and forced transition scenarios. In particular, for the free transition case an early stall takes place between 2 and 4 degrees, while for the forced transition case a standard polar is observed.

The auxiliary input data for the application of the rotational augmentation model are taken from Ref. [43]. By means of a procedure similar to that described in the previous subsections, the $a$ parameter for the CH RAM with the best fitting for the CFD unsteady RANS results can be determined. Depending on the choice for the input two-dimensional lift polar curve different scenarios can be investigated. In Fig. 16 the application of the fit procedure for the proposed alternative parametric formulation is shown. The fitting procedure results in $a_{\text {lam }} \approx 0.7067$ and $a_{t u r} \approx-1.3578$ for, respectively, the laminar and turbulent input lift polar case. Moreover, a better approximation is possible when the laminar experimental lift polar curve is used as input for the fitted CH RAM. The effect of the Prandtl root correction for this 


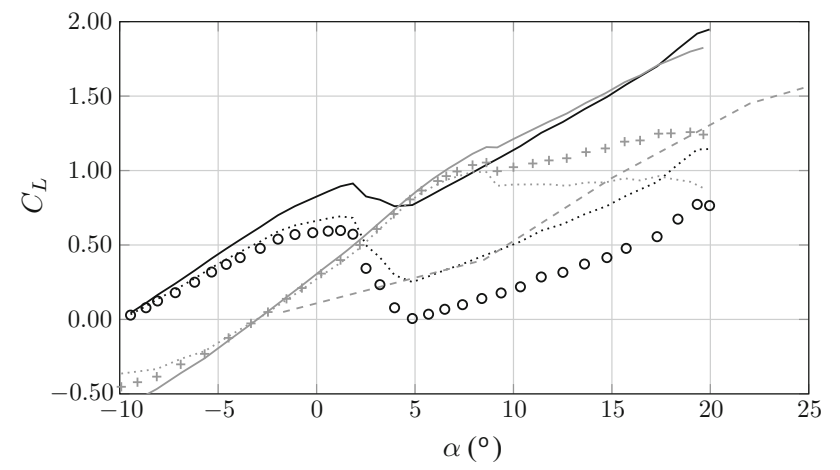

Fig. 16 Application of baseline and $a$-parameter based fitted CH RAM for the lift coefficient of the airfoil DU-00-W-401. Experimental results from Ref. [42] for a Reynolds number equal to $3 \times 10^{6}$. CFD results from Ref. [25] for the same airfoil located at the $25 \%$ of the blade length for the AVATAR rotor. The CH RAM is applied to both laminar and fully turbulent experimental two-dimensional lift polar curve. The fitting procedure results in $a_{\text {lam }} \approx 0.7067$ and $a_{t u r} \approx-1.3578$ for, respectively, the laminar and turbulent input lift polar case. Legend: experimental result with free transition (+), experimental result for forced transition at $2 \%$ suction side and $10 \%$ pressure side ( $\circ$ ), baseline CH RAM applied on free transition experimental polar (-), baseline CH RAM applied on forced transition experimental polar (-), fitted CH RAM applied on free transition experimental polar (.), fitted CH RAM applied on forced transition experimental polar (·), CFD unsteady RANS from Ref. [25] for a comparable airfoil section having the maximum relative thickness equal to $40 \%$ and located at the $25 \%$ of the blade length for the AVATAR rotor (- -)

section, equal to 0.9768 , does play a minor role in this case, leading to a value for the fitted $a$ parameter equal to 0.7216 .

Moreover, the correct capture of the lift polar curve identified by means of CFD simulation at the lowest inflow velocity speed value remains unattainable for the fitted $\mathrm{CH}$ RAM based on the laminar experimental lift polar curve. In fact, the lift polar point located at an AoA of about $-2^{\circ}$ lies closer to the turbulent lift polar curve. For this reason, further threedimensional numerical simulation in absence of rotation may serve to determine the lift polar basis on which the RAM is to be applied, thus, separating the different levels related to the two-dimensional data referring to infinite body from the two-dimensional data referring to finite body, and both the latter from the two-dimensional data referring to finite rotating body.

\section{Conclusions and outlook}

In this work the rotational augmentation effects are studied for developing an alternative parametric formulation based on the baseline version of Chaviaropoulos and Hansen [16]. The novel formulation is calibrated based on the results of CFD simulations.

Starting from validated simulation setups, the finiteness and rotational augmentation effects are estimated by com- paring rotating CFD results against two-dimensional polars corrected for tip and root effects prior to the application of RAM selected from the literature.

The best agreements for both lift and drag coefficients were found for the model of Chaviaropoulos and Hansen [16], mainly at inboard regions where the rotational augmentation effects are stronger.

In order to extend the good performance of this model to a broader range of operating conditions and blade locations, a an alternative parametric formulation was derived and presented, together with its interaction in conjunction with the application of tip and root corrections. Subsequently, an optimization procedure based on curve fitting led to the determination of the optimal values for the $a_{n}$ parameter, resulting in a piece-wise modeling dependent on inflow wind velocity, i.e. operating tip speed ratio, and span-wise position. The novel formulation results in improved lift and drag coefficients prediction in all considered cases. The optimal configuration including the correction for tip effects of Shen shows better agreements at the very tip of the blade. One key point is to verify the proposed alternative parametric formulation in terms of independence with respect to the choice of the wind turbine model. The authors have reported some preliminary observations concerning the applicability of the proposed procedure for larger wind turbine rotor blades for the virtual model of the AVATAR rotor. With this regard, further issues should be addressed in future works, namely those concerning the determination of the initial lift (and drag) polar curve to be used as input for the application of the RAM. In fact, the usage of thicker aerodynamic shapes in the inboard part of the blade introduces large deviations from the standard thin airfoil section lift polar characteristics at low angle of attacks. This circumstance may be explored by means of additional DES simulations in order to capture with better approximation the highly separated region [44], possibly by means of laminar-turbulent transition modeling, in order to determine the non-rotating flow condition at the inboard region of the blade. Once these open points are clarified the application of the alternative parametric RAM formulation to these thick inboard sections may be conducted with reduced uncertainty.

Acknowledgements Open Access funding provided by Projekt DEAL. This work was funded by the German Ministry of Economy in the framework of HighRe National project (Grant 03EE2001)

Open Access This article is licensed under a Creative Commons Attribution 4.0 International License, which permits use, sharing, adaptation, distribution and reproduction in any medium or format, as long as you give appropriate credit to the original author(s) and the source, provide a link to the Creative Commons licence, and indicate if changes were made. The images or other third party material in this article are included in the article's Creative Commons licence, unless indicated otherwise in a credit line to the material. If material is not included in the article's Creative Commons licence and your 
intended use is not permitted by statutory regulation or exceeds the permitted use, you will need to obtain permission directly from the copyright holder. To view a copy of this licence, visit http://creativecomm ons.org/licenses/by/4.0/.

\section{References}

1. United Nations Organization: Paris Agreement. https://treaties.un. org/Pages/ViewDetails.aspx?src=TREATY\&mtdsg_no=XXVII7-d\&chapter $=27 \&$ lang=_en\&clang=_en $(2016)$

2. Moccia, J., Arapogianni, A., Wilkes, J., et al.: Pure power: wind energy targets for 2020 and 2030. http://www.ewea.org/fileadmin/ files/library/publications/reports/Pure_Power_III.pdf (2009)

3. Sunak, Y., Höfer, T., Siddique, H., et al.: A GIS-based decision support system for the optimal siting of wind farm projects. https:// publications.rwth-aachen.de/record/660367 (2015)

4. Rahimi, H.: Validation and improvement of numerical methods for wind turbine aerodynamics : development of an engineering model for yawed conditions derived from computational fluid dynamics. Ph.D, Faculty of Physics. https://plus.orbis-oldenburg.de/ permalink/f/11df5a8/49GBVUOB_ALMA21238396660003501 (2018)

5. Schaffarczyk, A.: Understanding Wind Power Technology: Theory, Deployment and Optimisation. Wiley, New York (2014). https:// doi.org/10.1002/9781118701492

6. Rahimi, H., Martinez Garcia, A., Stoevesandt, B., et al.: An engineering model for wind turbines under yawed conditions derived from high fidelity models. Wind Energy 5(2-3), 85 (2018). https:// doi.org/10.1002/we. 2182

7. Thumthae, C., Chitsomboon, T.: Improved 3D stall delay model for wind turbine design. In: The 12th Americas Conference on Wind Engineering (12ACWE), June 16-20. https://depts.washington. edu/uwconf/12acwe/schedule_details.html\#w2 (2013)

8. Madsen, H.A., Rasmussen, F.: Derivation of three dimensional airfoil data on the basis of experiment and theory. In: Proceedings of Wind Power, vol. 88, pp. 166-174. https://www.worldcat. org/title/windpower-98-proceedings-april-27-may-1-1998holiday-inn-select-convention-center-bakersfield-californiaannual-conference-exhibition-of-the-american-wind-energyassociation/oclc/41282180\&referer=brief_results (1988)

9. Lindenburg, C.: Investigation into Rotor Blade Aerodynamics: analysis of the stationary measurements on the UAE phase-VI rotor in the NASA-Ames wind tunnel. https://publications.tno.nl/ publication/34628279/4XkI02/c03025.pdf

10. Dowler, J.L., Schmitz, S.: A solution-based stall delay model for horizontal-axis wind turbines. Wind Energy 18(10), 1793-1813 (2015). https://doi.org/10.1002/we.1791

11. Schreck, S.J., Robinson, M.C.: Horizontal axis wind turbine blade aerodynamics in experiments and modeling. IEEE Trans. Energy Convers. 22(1), 61-70 (2007). https://doi.org/10.1109/TEC.2006. 889620

12. Gonzalez, A., Munduate, X.: Three-dimensional and rotational aerodynamics on the NREL Phase VI wind turbine blade. J. Solar Energy Eng. 130(3), 031008 (2008). https://doi.org/10.1115/1. 2931506

13. Bangga, G.: Three-dimensional flow in the root region of wind turbine rotors. Ph.D. Thesis, University of Stuttgart, Stuttgrat, Germany. http://www.uni-kassel.de/upress/online/OpenAccess/9783-7376-0536-6.OpenAccess.pdf (2018)

14. Du, Z., Selig, M.: The effect of rotation on the boundary layer of a wind turbine blade. Renew. Energy 20(2), 167-181 (2000). https:// doi.org/10.1016/S0960-1481(99)00109-3

15. Snel, H., Houwink, R., Bosschers, J.: Sectional prediction of lift coefficients on rotating wind turbine blades in stall. https:// www.worldcat.org/title/sectional-prediction-of-lift-coefficientson-rotating-wind-turbine-blades-in-stall/oclc/60178514

16. Chaviaropoulos, P.K., Hansen, M.O.L.: Investigating threedimensional and rotational effects on wind turbine blades by means of a quasi-3D Navier-Stokes solver. J Fluids Eng 122(2), 330 (2000). https://doi.org/10.1115/1.483261

17. Corrigan, J. J., Schillings, J.J.: Empirical Model For Stall Delay Due To Rotation. https://vtol.org/store/product/empirical-modelfor-stall-delay-due-to-rotation-7693.cfm (1994)

18. Tangler, J.L., Selig, M.S.: An Evaluation of an Empirical Model for Stall Delay due to Rotation for HAWTS. https://www.nrel.gov/ docs/legosti/old/23258.pdf

19. Bak, C., Johansen, J., Andersen, P.B.: Three-dimensional corrections of airfoil characteristics based on pressure distributions. In: European Wind Energy Conference \& Exhibition (EWEC). http://citeseerx.ist.psu.edu/viewdoc/download?doi=10. 1.1.562.9634\&rep=rep1\&type $=$ pdf (2006)

20. Dumitrescu, H., Cardoş, V., Dumitrache, A.: Modelling of inboard stall delay due to rotation. J. Phys. Conf. Ser. 75, 012022 (2007). https://doi.org/10.1088/1742-6596/75/1/012022

21. Bangga, G.S., Lutz, T., Krämer, E.: An examination of rotational effects on large wind turbine blades. EAWE. https://www. eera-avatar.eu/fileadmin/avatar/user/Bangga_EAWE_2015_ FullPaper_RG.pdf (2015)

22. Herráez, I., Daniele, E., Schepers, J.G.: Extraction of the wake induction and angle of attack on rotating wind turbine blades from PIV and CFD results. Wind Energy Sci. 3(1), 1-9 (2018). https:// doi.org/10.5194/wes-3-1-2018

23. Johansen, J., Sørensen, N.N.: Aerofoil characteristics from 3D CFD rotor computations. Wind Energy 7(4), 283-294 (2004). https:// doi.org/10.1002/we.127

24. Rahimi, H., Schepers, J.G., Shen, W.Z., et al.: Evaluation of different methods for determining the angle of attack on wind turbine blades with CFD results under axial inflow conditions. Renew. Energy 125, 866-876 (2018). https://doi.org/10.1016/j. renene.2018.03.018

25. Bangga, G., Lutz, T., Jost, E., et al.: CFD studies on rotational augmentation at the inboard sections of a $10 \mathrm{MW}$ wind turbine rotor. J. Renew. Sustain. Energy 9(2), 023304 (2017). https://doi. org/10.1063/1.4978681

26. Bangga, G., Lutz, T., Krämer, E.: Root flow characteristics and $3 \mathrm{D}$ effects of an isolated wind turbine rotor. J. Mech. Sci. Technol. 31(8), 3839-3844 (2017). https://doi.org/10.1007/s12206017-0728-6

27. Tian, W., Ozbay, A., Wang, X.D., et al.: Experimental investigation on the wake interference among wind turbines sited in atmospheric boundary layer winds. Acta Mech. Sin. 33(4), 742-753 (2017). https://doi.org/10.1007/s10409-017-0684-5

28. Rahimi, H., Daniele, E., Stoevesandt, B., et al.: Development and application of a grid generation tool for aerodynamic simulations of wind turbines. Wind Eng. 40(2), 148-172 (2016). https://doi. org/10.1177/0309524X16636318

29. Menter, F.R.: Two-equation eddy-viscosity turbulence models for engineering applications. AIAA J. 32(8), 1598-1605 (1994). https://doi.org/10.2514/3.12149

30. Spalart, P.R., Rumsey, C.L.: Effective inflow conditions for turbulence models in aerodynamic calculations. https://ntrs.nasa.gov/ archive/nasa/casi.ntrs.nasa.gov/20070035069.pdf (2007)

31. Norris, S.E.: A parallel Navier-Stokes solver for natural convection and free surface flow. Ph.D. Thesis, University of Sydney, Sydney, Australia. https://ses.library.usyd.edu.au/handle/2123/376 (2009)

32. The University of Oldenburg: The HPC User Wiki of the University of Oldenburg. https://uol.de/en/school5/sc/high-perfomancecomputing/faq-frequently-asked-questions (2020)

33. Hand, M.M., Simms, D.A., Fingersh, L.J., et al: Unsteady Aerodynamics Experiment Phase VI: Wind Tunnel Test Configura- 
tions and Available Data Campaigns. Tech. Rep. NREL/TP-50029955, NREL, Golden, Colorado, USA. https://www.nrel.gov/ docs/fy02osti/29955.pdf (2001)

34. Butterfield, C.P., Musial, W.P., Simms, D.A.: Combined Experiment Phase I: Final Report. Tech. Rep. NREL/TP-257-4655, NREL, Golden, Colorado, USA. https://www.nrel.gov/docs/ legosti/old/4655.pdf (1992)

35. Rahimi, H., Medjroubi, W., Stoevesandt, B., et al.: Navier-Stokesbased predictions of the aerodynamic behaviour of stall regulated wind turbines using OpenFOAM. Prog. Comput. Fluid Dyn. Int. J. 16(6), 339 (2016). https://www.inderscienceonline.com/doi/abs/ 10.1504/PCFD.2016.080054

36. Sørensen, N.N., Michelsen, J.A., Schreck, S.: Navier-Stokes predictions of the NREL phase VI rotor in the NASA Ames $80 \mathrm{ft} \times$ $120 \mathrm{ft}$ wind tunnel. Wind Energy 5(2-3), 151-169 (2002). https:// doi.org/10.1002/we.64

37. Guntur, S., Sørensen, N.N.: An evaluation of several methods of determining the local angle of attack on wind turbine blades. J. Phys. Conf. Ser. 555, 012045 (2014). https://doi.org/10.1088/ 1742-6596/555/1/012045

38. Glauert, H.: Airplane propellers. In: Durand, W.F. (ed.) Aerodynamic Theory. Springer, Berlin (1935). https://doi.org/10.1007/ 978-3-642-91487-4_3
39. Shen, W.Z., Zhu, W.J., Sørensen, J.N.: Study of tip loss corrections using CFD rotor computations. J. Phys. Conf. Ser. 555, 012094 (2014). https://doi.org/10.1088/1742-6596/555/1/012094

40. Schepers, J.G.: AVATAR project final report: Advanced aerodynamic modelling, design and testing for large rotor blades. https://www.eera-avatar.eu/fileadmin/avatar/user/AVATAR_ final_report_v26_2_2018.pdf

41. Li, S., Li, Y., Yang, C., et al.: Experimental and numerical investigation of the influence of roughness and turbulence on LUT airfoil performance. Acta Mech. Sin. 35(6), 1178-1190 (2019). https:// doi.org/10.1007/s10409-019-00898-3

42. van Rooij, R.P.J.O.M., Timmer, W.A.: Roughness sensitivity considerations for thick rotor blade airfoils. ASME J. Solar Energy Eng. 125(4), 468-478 (2003). https://doi.org/10.1115/1.1624614

43. Reference Blade Design: AVATAR Deliverable D1.2. http:// www.eera-avatar.eu/fileadmin/avatar/user/2015_01_30_D1_2_ AVATAR_Reference_Blade_Design rev_03_09.pdf

44. Zheng, W., Yan, C., Liu, H., et al.: Comparative assessment of SAS and DES turbulence modeling for massively separated flows. Acta Mech. Sin. 32(1), 12-21 (2016). https://doi.org/10.1007/s10409015-0505-7 